\title{
Análisis del programa Prospera sobre la disminución de la pobreza en México entre 1997-2017
}

Diana Jazmín Meza Maldonado*

\section{Resumen}

$\mathrm{C}^{\text {lobjetivo de este trabajo es analizar la contribución del progra- }}$ Cma Prospera para la disminución de la pobreza en México durante el período 1997-2017. Para esto, se utilizó una metodología descriptiva y analítica con diferentes fuentes de consulta de artículos, libros, páginas oficiales de organismos y tesis. Los resultados muestran que si bien el programa Prospera ha traído beneficios a las familias mexicanas como el incremento de los becarios en materia de educación, en más de un millón de estos beneficios no han podido consolidarse. El número de pobres aumentó a casi 2 millones, también se observó una disminución del padrón de familias beneficiarias de 75 entre 2014 y 2017. El gobierno actual debería implementar programas afines a las necesidades de la población vulnerable y desarrollar mecanismos para su efectiva aplicación.

\author{
Palabras clave \\ Programas Sociales; Desigualdad; CONEVAL; \\ México; Política Pública
}

\footnotetext{
* Doctora en Dirección de Organizaciones por la Universidad del Distrito Federal, Maestra en Administración Pública por la Universidad del Valle de México con Especialidad en Gestión Pública por la FLACSO México, y Licenciada en Derecho por la Universidad Autónoma de Nayarit. Actualmente se desempeña como Encargada de la Dirección de Sistema de Información en la Coordinación Nacional de Becas para el Bienestar Benito Juárez, Secretaría de Educación Pública. diana_mezam@hotmail.com.
} 
Fecha de recepción:

Marzo de 2020
Fecha de aceptación:

junio de 2020

Analysis of the Prospera program on the reduction of poverty in Mexico from 1997-2017

Key words

Social Programs; Inequality; CONEVAL; Mexico; Public Policy.

Final submission:

March 2020
Acceptance:

June 2020

\section{Abstract:}

The objective of this paper is to analyse the contribution of the Prospera program to reduce poverty in Mexico during the period 1997-2017. To do so, a descriptive and analytical methodology was used with different sources such as articles, books, official pages of organizations and thesis. The results show that while the prospering program has brought benefits to Mexican families such as an increase in education fellows, more than one million, these benefits have not been consolidated. The number of poor increased of almost 2 million, there was also a decrease in the register of beneficiary families of 75 between 2014 and 2017. The present government should implement programs related to the needs of the vulnerable population and develop mechanisms for their effective application.

\section{Introducción}

La pobreza es una noción multidimensional razón por la cual atribuirle una sola definición es tema de mucho riesgo, dado que cada país tiene características propias que cambian o se interpretan de manera distinta en otros países. De cualquier forma, se utilizará la definición de un organismo especialista del tema. Para el Banco Mundial ${ }^{1}$ la pobreza es la incapacidad para alcanzar un nivel de vida mínimo.

Esta institución establece un nivel basado en el consumo integrado por dos factores. El primero se refiere a: "el gasto necesario para acceder a un estándar mínimo de nutrición y otras necesidades muy básicas" y el segundo a: "una cantidad que varía de un país a 
otro y que refleja el costo que tiene la participación en la vida diaria de las sociedades"2 . La pobreza tiene repercusiones en la asignación eficiente de los recursos puesto que las personas en esa situación no pueden desarrollar todo su verdadero potencial. ${ }^{3}$

Ahora bien, para abatir el dilema de la pobreza, los gobiernos diseñan políticas públicas focalizadas como en el caso mexicano donde han implementados una serie de acciones con la finalidad de mejorar los estándares de vida de la población.

Una de esas políticas es el Programa de Educación, Salud y Alimentación, actualmente conocido con el nombre de PROSPERA, creado en 1997, ${ }^{4}$ a fin de ejecutar a través de políticas públicas, acciones que permitan que los ciudadanos gocen del bienestar que sus derechos les otorgan.

Sin embargo, esas directrices no han traído el resultado esperado. Para el Consejo Nacional de Evaluación de la Política de Desarrollo Social, 52,425,887 de personas en México se encontraban en situación de pobreza en el 2018. Asimismo, ese mismo año se estimaron 9,310,153 personas en pobreza extrema, es decir, el $7.4 \%$ de la población mexicana, razón por la que, el nivel de pobreza alimentaria continúa siendo uno de los grandes desafíos en México.5

Adicionalmente, el organismo mencionado señala que en el 2010 el $18.8 \%$ de la población total generaba un ingreso menor al valor de la línea de pobreza alimentaria. Esta línea de pobreza corresponde al costo de adquirir una serie de bienes alimenticios bá-

2 Fernández, Andrés. Martínez, Rodrigo. Carrasco, Ignacio y Palma, Amalia. "Impacto social y económico de la malnutrición. Un modelo de análisis y estudio piloto en Chile, el Ecuador y México”. Programa Mundial de Alimentos, CEPAL, Naciones Unidas. 2017., p. 26.

3 Campos Vázquez, Raymundo et al. "La relación entre crecimiento económico y pobreza en México”, Investigación Económica, 75, no. 298, (2016), https://www.redalyc.org/pdf/601/60148183003.pdf (consultado el 25 de febrero de 2020).

4 Este programa ha tenido varios nombres a lo largo de su establecimiento: Progresa de 1997-2002, Oportunidades de 2002-2014 y Prospera de 2014-2018.

5 CONEVAL (Consejo Nacional de Evaluación de la Política de Desarrollo Social), Estrategias de coordinación interinstitucional para la política de desarrollo social en México, Coneval https://www.coneval.org.mx/Evaluacion/IEPSM/Documents/ Documento-completo-40-A-de-estrategias.pdf. 
sicos, por lo que refleja que casi uno de cada cinco habitantes aún carece de los recursos para satisfacer necesidades nutricionales mínimamente adecuadas. ${ }^{6}$

Lo anterior preocupa a pesar de haberse hecho esfuerzos en programas. De allí surge el cuestionamiento siguiente: ¿cuál ha sido la contribución del programa PROSPERA en la pobreza en México desde su creación hasta 2017? El objetivo de este trabajo es analizar la contribución de este programa para la disminución de la pobreza en México.

El trabajo presentará, en primera instancia, un panorama de diferentes modelos de evaluación de la pobreza, después la política de Estado en materia de pobreza; posteriormente la política pública aplicada en materia social y por ende las políticas sociales, de la cual el tema de interés es el programa PROSPERA. Finalmente se redacta la conclusión de este documento.

\section{Modelo de evaluación}

En la actualidad, para medir la pobreza se emplean los métodos de recolección de información (encuestas). Sin embargo, estos métodos, son amenazados por el bajo costo de acceder a datos masivos (Big Data), lo que permite, generar datos a toda hora y de diferentes formas, desde información espacial e información geográfica hasta llamadas a celulares. En temas de pobreza, estos métodos permiten medir una amplia gama de indicadores.

De acuerdo al Banco Mundial, el acceso a este tipo de información hace replantear el futuro de la información estadística. La medición de la pobreza transita hacia el uso de información masiva poco costosa, pero de gran valor informativo. Los gobiernos de los llamados países en desarrollo aún no han adoptado estas medidas para medir el bienestar de la población de manera oficial. ${ }^{7}$

\footnotetext{
6 Székely Pardo, Miguel et al. "Pobreza alimentaria y desarrollo en México", Trimestre económico. 81 no. 321 (2014).

7 Tejerina, Luis et al. América Latina y el Caribe debería aprovechar los datos masivos para medir la pobreza. https://blogs.iadb.org/efectividad-desarrollo/es/america-latina-deberia-aprovechar-los-datos-masivos-para-medir-la-pobreza/
} 
Las Naciones Unidas han creado el laboratorio Global Pulse, a fin de aprovechar las ventajas que ofrecen los datos masivos para explorar y dar seguimiento al cumplimento de los Objetivos de Desarrollo Sostenible a un costo menor que lo empleado en los métodos tradicionales. ${ }^{8}$

El (2005) ${ }^{9}$ cuenta con un Sistema de Información de Indicadores Sociales y Equidad denominado EQxIS. Este sistema utiliza información de las encuestas de los hogares (Mejoramiento de las Encuestas y la Medición de las Condiciones de Vida en América Latina y el Caribe MECOVI) y estima indicadores sociales, los cuales miden el avance hacia el logro de los Objetivos de Desarrollo del Milenio fijados en el marco de las Naciones Unidas.

Con los datos de EQxIS se puede saber, por ejemplo:

- Si la situación de los diferentes grupos de la población respecto al promedio nacional es equitativa.

- Si los avances o retrocesos afectaron o beneficiaron por igual a la población de menores y mayores ingresos, o a la de las áreas urbanas y rurales.

- Si los países han avanzado a diferente ritmo.

Esto significa que, en un tiempo no muy lejano, la medición y seguimiento de los indicadores de pobreza a nivel local se realizarán usando la información masiva; es decir, la información masiva dejará de ser novedad para convertirse en una realidad y así brindar información de encuestas de hogares que sea oportuna, comparable, de buena calidad, de fácil acceso, y con una mayor cobertura geográfica y temática.

\footnotetext{
8 Idem.

9 Banco Interamericano de Desarrollo, "BID lanza indicadores sociales y de equidad para medir avances y problemas en logro Objetivos de Desarrollo del Milenio", Banco Interamericano de Desarrollo. https://www.iadb.org/es/noticias/comunicados-de-prensa/2005-10-28/bid-lanza-indicadores-sociales-y-de-equidad-para-medir-avances-y-problemas-en-logro-objetivos-de-desarrollo-del-milenio $\% 2$ c1 188 . html.
} 
Finalmente, a nivel nacional, el Consejo Nacional de Evaluación de la Política de Desarrollo Social (Coneval en adelante), en la metodología para la medición multidimensional de la pobreza en México (2018), menciona que, en la medición de la pobreza, utiliza indicadores que le permite identificar y medir la pobreza en sus diferentes clasificaciones, para ello, el Coneval y el Instituto Nacional de Estadística y Geografía (INEGI en adelante) iniciaron una colaboración interinstitucional a fin de generar fuentes de información en un solo instrumento que capte la totalidad de los indicadores de medición basados en la información de las Condiciones Socioeconómicas de la Encuesta Nacional de Ingresos y Gastos de los Hogares (ENIGH) para realizar la medición de la pobreza con apego a lo dispuesto en la Ley General de Desarrollo Social. ${ }^{10}$

\subsection{Políticas de Estado}

Las políticas de Estado son aquellas políticas que integran las estrategias centrales de un país. Son políticas a largo plazo que siguen un proyecto definido sobre temas como educación, infraestructuras básicas, salud, gasto público, entre otros. Las políticas de Estado no varían a pesar del color político o ideología de cada gobierno; es decir, van más allá de una estrategia de políticas sexenales, tienen un valor estratégico que los gobiernos sea cual sea su color deben seguir en pro de la continuidad y estabilidad del país.

Una política de Estado no puede pensarse como un programa unilateral, rígido, definido previamente, sino como el resultado complejo y cambiante de la combinación de fuerzas políticas, equilibrios sociales y tendencias históricas y culturales. ${ }^{11}$ Por otra parte, "Una política de Estado, en la medida que trasciende a los

${ }^{10}$ CONEVAL (Consejo Nacional de Evaluación de la Política de Desarrollo Social), "Estrategias de coordinación interinstitucional para la política de desarrollo social en México", https://www.coneval.org.mx/Evaluacion/IEPSM/Documents/Documento-completo-40-A-de-estrategias.pdf.

${ }^{11}$ Natanson José. “¿Qué es, en realidad, una política de Estado?”, (2008), https:// www.pagina12.com.ar/diario/elpais/1-106918-2008-06-29.html. 
partidos y a las ideologías debería ser producto del consenso o de un gran acuerdo nacional que ponga por delante el interés del conjunto de la Nación"; ${ }^{12}$ y para construir políticas de Estado, se debe partir del consenso y de la confrontación y requieren un Estado capaz de impulsar y coordinar debates colectivos propios de la democracia. ${ }^{13}$

En suma, el Estado es el único que puede definir y desarrollar políticas de Estado que permitan redireccionar el rumbo del país a fin de satisfacer las necesidades de su ciudadanía y consolidarse como un Estado establece y continuo. Las políticas de Estado representan el plan nacional que sigue el gobierno en su dirección sexenal; deben seguirse al pie de la letra independientemente de la ideología política en el poder y para ser eficaces deben adaptarse y transformarse a los cambios culturales, históricos y sociales, entre otros.

\subsection{Políticas Públicas}

Hablar de políticas públicas inspira a pensar en las tareas que cada gobierno define para atender las Banco Mundial necesidades de sus ciudadanos, los lineamientos que implementa cada nación, deben considerar en su definición las características emitidas por los organismos internacionales con la finalidad de que sea específica al problema detectado, es decir, si se habla de pobreza, la política pública que se implemente para reducirla, debe considerar las variables conceptualizadas por la Comisión Económica para América Latina y el Caribe (CEPAL en adelante), el Banco Interamericano de Desarrollo (BID en adelante), el Programa de las Naciones Unidas para el Desarrollo (PNUD en adelante) entre otros organismos y especialistas en la materia, a fin de que la acción que se realice incida positivamente en las carencias de las personas y, sobre todo, dé cumplimiento a las obligaciones que

\footnotetext{
12 Rulli, Jorge. "Producir soja como política de Estado”. Ecoportal.net. 2006. http:// www.ecoportal.net/Temas_Especiales/Transgénicos/Producir_soja_como_politica_de_Estado.

${ }^{13}$ Romero Luis Alberto. "Cómo crear políticas de Estado". Perfil.com. http:// www. perfil.com/contenidos/2010/08/21/noticia_0034.html.
} 
dichos organismos internacionales imponen en pro de los préstamos internacionales.

En este orden de ideas, "Las políticas públicas son aquellos cursos de acción que el gobierno decide tomar frente a problemas específicos. Se dice que es aquello que el gobierno hace o deja de hacer y que atiende fundamentalmente un problema o situación determinada" ${ }^{14}$ Por otro lado la política pública es la ciencia de la acción administrativa, que se constituye en: El análisis de las políticas se ha constituido, en particular en Estados Unidos, como una ciencia de la acción, como una contribución de los expertos a las decisiones de las autoridades gubernamentales. ${ }^{15}$

"La formulación de políticas públicas es una tarea compleja. Concretar una determinada "reforma de política" es un proceso que involucra a muchos actores a través de varias fases del proceso de formulación de políticas, requieren acciones específicas de parte de los agentes económicos y sociales y por lo tanto, se necesitan diversas formas de cooperación, y confianza en la durabilidad de la política, y otras características semejantes a efecto de que los resultados sean efectivos". ${ }^{16}$

En México, la inserción de las políticas públicas en el campo disciplinario y de investigación académica, así como en el ámbito social y político, está ligada al proceso de cambios en la política de nuestro país tendiente a la democratización, que inició con la crisis política y económica en los años ochenta. ${ }^{17}$

${ }^{14}$ Jiménez Guzmán, Rodolfo, Las politicas públicas, gerencia y gestión pública moderna, panaceas de racionalidad y eficiencia, o nuevas tecnologías de gobierno. UAM: Revista casa del tiempo. México: 1999)., p. 130.

${ }^{15}$ Cantú Herrera, Jorge Raúl y Chiatchoua Cesaire (2018). Generación de políticas públicas en materia fiscal para la permanencia de las unidades económicas en México (20052017). Revista. ConCiencia. Vol.8, págs. 30-45. Consultado el 8 de junio de 2020 en: https://fup.edu.co/wp-content/uploads/2019/06/conciencia_8_articulo_3.pdf

${ }^{16}$ Banco Interamericano de Desarrollo, "BID lanza indicadores sociales y de equidad para medir avances y problemas en logro Objetivos de Desarrollo del Milenio" Banco Interamericano de Desarrollo, https://www.iadb.org/es/noticias/comunicados-de-prensa/2005-10-28/bid-lanza-indicadores-sociales-y-de-equidad-para-medir-avances-y-problemas-en-logro-objetivos-de-desarrollo-del-milenio\%2c1188.html.

${ }^{17}$ Aguilar Villanueva, Luis. "Recepción y desarrollo de la disciplina de Política Pública en México. Un estudio introductorio Sociológica”, 19, no. 54 (2004): 15-37. 
Inicialmente, el Estado mexicano se resistía a implementar las políticas públicas durante el periodo conocido como Sustitución de Importaciones, toda vez que estaban acostumbrados a que el poder presidencial y los grupos minoritarios de la elite tomarán decisiones dentro de la administración pública sin dar cuentas a nadie sobre su actuar y mucho menos sobre los resultados. Ello, obstaculizaba el quehacer de la administración, así que decidieron de origen que, en lugar de llamar "políticas públicas", se utilizara el término "políticas de gobierno" o "políticas gubernamentales" a fin de que el interés privado prevaleciera sobre el público.

Se concluye que, a través de las políticas públicas, el gobierno identifica la problemática de la ciudadanía, la analiza, decide las acciones a realizar y las lleva a la práctica a través de la administración pública. Aunque algunas veces, estas acciones van dirigidas con miras al clientelismo electoral y no enfocadas en el bienestar social.

\subsection{Políticas Sociales}

La relación entre Estado, política pública y política social se refiere a, que el Estado es el ente que ejerce la autoridad ante la sociedad (individuos y organizaciones) en un territorio determinado a través de su organización, sus niveles de gobierno (federal, estatal, municipal) y basado en políticas, se vincula con la sociedad y la economía a través de políticas públicas.

La política social es definida a menudo en términos de servicios sociales como la educación, la salud, o la seguridad social. Sin embargo, "la política social incluye mucho más: distribución, protección y justicia social. La política social consiste en situar a los ciudadanos en el núcleo de las políticas públicas, ya no mediante el suministro de asistencia social residual, sino incorporando sus necesidades y voz en todos los sectores" ${ }^{18}$

Las políticas públicas a través de las políticas sociales deben garantizar que la economía crezca de manera estable y sostenida, la

${ }^{18}$ Ortiz, Isabel. "Política Social”. 2007. Documento de las Estrategias nacionales de Desarrollo, Naciones Unidas, https://esa.un.org/techcoop/documents/socialpolicy_spanish.pdf 
inversión social en mayor cantidad y calidad contribuye al crecimiento de la economía, si crece la economía crece la calidad de vida de la población.

La Política Social por su parte, es la encargada de la implementación de las estrategias nacionales de desarrollo (políticas públicas); es decir, a través de la política social el Estado se relaciona con la sociedad para asegurar los bienes y servicios públicos, superar el círculo vicioso de la pobreza, y crear un círculo virtuoso en el que el desarrollo humano y el empleo permitan el crecimiento económico del país. ${ }^{19}$

La política social es la estrategia del Estado para proporcionar bienes y servicios a sus ciudadanos en aras de elevar los niveles de vida proporcionando calidad y bienestar.

Si bien es cierto que las políticas sociales son el medio para atender las necesidades de la población, su aplicación en algunos casos lleva a la exclusión social; por ello, se deben implementar políticas sociales encaminadas a la superación de la pobreza y al bienestar común, justo e igualitario.

\subsection{Programas sociales: El caso de PROSPERA}

El programa PROSPERA, implementado en México en 1997 con el nombre de PROGRESA, ha sido el programa líder en cuanto a transferencias condicionadas, cuyo objetivo general es el de "Contribuir a fortalecer el cumplimiento efectivo de los derechos sociales que potencien las capacidades de las personas en situación de pobreza, a través de acciones que amplíen sus capacidades en alimentación, salud y educación, y mejoren su acceso a otras dimensiones del bienestar". ${ }^{20}$

La población a la que va dirigido son los hogares con ingreso per cápita estimado inferior a la Línea de Bienestar Mínimo

${ }_{19}$ Ortiz, Isabel. "Política Social". 2007. Documento de las Estrategias nacionales de Desarrollo, Naciones Unidas. https://esa.un.org/techcoop/documents/socialpolicy_spanish.pdf

${ }^{20}$ Secretaria de Gobernación. "Acuerdo por el que se emiten las Reglas de Operación de PROSPERA programa de Inclusión Social, para el ejercicio fiscal 2018”, Secretaría de Gobernación https://dof.gob.mx/nota_detalle.php?codigo=5509738\&fecha=29/12/2017 (Consultado el 1-1-2020). 
(LBM), cuyas condiciones socioeconómicas y de ingreso impiden desarrollar las capacidades de sus integrantes, específicamente en materia de alimentación, salud y educación.

Las familias elegibles para ingresar o reingresar al Programa son aquellas cuyo ingreso mensual per cápita estimado es menor a la Línea de Bienestar Mínimo ajustada (LBMa). Una vez identificadas las familias elegibles, el Programa da prioridad para su atención, a las siguientes:

- Hogares con integrantes menores a 22 años.

- Hogares con mujeres en edad reproductiva.

- Hogares con integrantes con alguna discapacidad.

Los apoyos otorgados consisten en los siguientes rubros:

En educación: becas educativas a cada uno de los niños, niñas y jóvenes menores de 18 años integrantes de las familias beneficiarias que cursen y concluyan sus estudios de Educación Básica y Media Superior; apoyo para la adquisición de útiles escolares; apoyo para Jóvenes con Prospera; acceso a ex becarios para garantizar su continuidad educativa a estudios de nivel superior; becas para estudios de nivel superior y para jóvenes con discapacidad y nuevas modalidades educativas como la no escolarizada, educación técnica y formación para el trabajo.

En alimentación: apoyo monetario mensual a las familias beneficiarias con la finalidad de contribuir a la compra y diversificación de sus alimentos; promoción de la nutrición y alimentación saludable para los niños y las niñas menores de 5 años, las mujeres embarazadas y en periodo de lactancia; suplementos alimenticios para complementar su dieta diaria; adquirir a un bajo costo productos lácteos con un alto valor nutricional en las lecherías Liconsa.

En salud: Acceso al Paquete Básico Garantizado de Salud y su migración progresiva a las 27 intervenciones del Catálogo Universal de Servicios de Salud (CAUSES en adelante); Talleres para el Autocuidado de la Salud, atención médica a cada integrante de la familia beneficiaria; prevención y atención a la mala nutrición; apoyos para adultos mayores; apoyo para integrantes de cero a nueve años; apoyo complementario para la alimentación; afilia- 
ción efectiva al Seguro Popular; incorporación de niños menores de cinco años al Seguro Médico Siglo XXI.

Inclusión laboral: se apoya para que, a través del Servicio Nacional del Empleo se facilite la incorporación de las familias beneficiarias al ámbito laboral.

Inclusión productiva: se tendrá acceso prioritario a 15 programas federales con salidas productivas, para incrementar su productividad y favorecer la generación de ingresos autónomos y sostenibles: Programa de Apoyo para la Productividad de la Mujer Emprendedora; Programa de Apoyo a Jóvenes para la Productividad de Futuras Empresas Rurales; Programa de Productividad y Competitividad Agroalimentaria, Programa Opciones Productivas; Programa del Fondo Nacional para el Fomento de las Artesanías; Fondo para el Apoyo a Proyectos Productivos en Núcleos Agrarios; Programa de Fomento a la Agricultura; Componente Acceso al Financiamiento Productivo y Competitivo; Programa Integral de Desarrollo Rural; Programa para el Mejoramiento de la Producción y Productividad Indígena; Programa de Fomento a la Economía Social; Bécate; Fomento al Autoempleo, el Fondo Nacional del Emprendedor y Programa para la Constitución y Operación de Unidades y Promoción de Crédito de Garantías Líquidas y Reducción de Costos de Acceso al Crédito.

Inclusión financiera: Esquema de acciones financieras específicas para las y los beneficiarios del Programa, con el objetivo de ofrecer herramientas adicionales que les permitan reducir la brecha de inclusión financiera y contribuir a mejorar su calidad de vida.

La titular beneficiaria, que generalmente es la madre de familia o quien se hace cargo del hogar, deberá cumplir junto con la familia corresponsabilidades en salud y educación para poder recibir los apoyos monetarios.

Las corresponsabilidades en salud con las que se deberán cumplir son: registrar a toda la familia a la unidad de salud que le corresponde, que cada miembro de familia acuda a las citas médicas y cumplan con la asistencia a los talleres comunitarios para el autocuidado de la salud. Las corresponsabilidades que las y los bene- 
ficiarios deben cumplir en educación son: inscribir a sus hijos en educación primaria, secundaria y media superior en escuelas autorizadas por el Programa y la asistencia regular de los becarios a clases.

\section{Impactos del programa Prospera en la sociedad mexicana}

El punto central de este documento es ¿Cuáles son los efectos del programa PROSPERA sobre la erradicación de la pobreza en México? Por eso, en el marco de un proyecto de colaboración entre la coordinación nacional de Prospera (Programa de Inclusión Social) y la Universidad Iberoamericana, en México, se llega a la conclusión que de 2010 a 2014, hubo una reducción en la carencia relacionada con el rezago educativo. ${ }^{21}$

Gráfica 1: Evolución de los becarios integrantes de las familias beneficiarias atendidos por Prospera al inicio de los ciclos escolares 1997-2017.

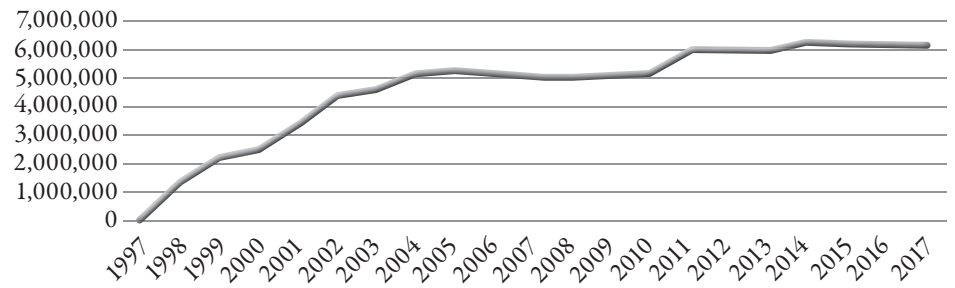

Fuente: Elaboración propia con base en Prospera, Programa de Inclusión Social, 2019.

La gráfica 1 muestra un incremento de los becarios integrantes de las familias beneficiarias de los programas entre 1997 y 2017 a pesar de una caída entre 2008 y 2009 . Se observa entre 2010 y 2014, un aumento del número de becarios que residen en hogares con rezago educativo entre la población PROSPERA (de $5,194,228$ de becarios a 6,194,542; es decir, $19.25 \%$ de crecimiento), en comparación con la disminución que experimenta la población no beneficiaria (de 19.7\% a 17.8\%). Más adelante se observa

${ }^{21}$ Arenas, Erika. Teruel, Graciela et al. "Resumen de Políticas PROSPERA Programa de Inclusión Social: Combate a la pobreza con perspectiva de género", PROSPERA-SEDESOL,https://www.gob.mx/cms/uploads/attachment/file/225888/ Documento_de_pol_ticas_sobre_g_nero.pdf 
un estancamiento de las mismas, es decir, de 6,194,542 de becas a 6,144,165 becarios, lo que indica una disminución de 50,377 becas.

Entre los beneficiarios de Prospera, la gráfica 2 muestra en promedio un crecimiento del número entre 1997 y 2014. Sin embargo, después de 2014, se observa una disminución de los beneficiarios, este corresponde a una reducción de casi $10 \%$ de los integrantes de familias beneficiarias; es decir, pasó de 29,728,293 en 2014 a 27,029,543 en 2017.

Gráfica 2. Integrantes de familias beneficiarias 2000- 2017.

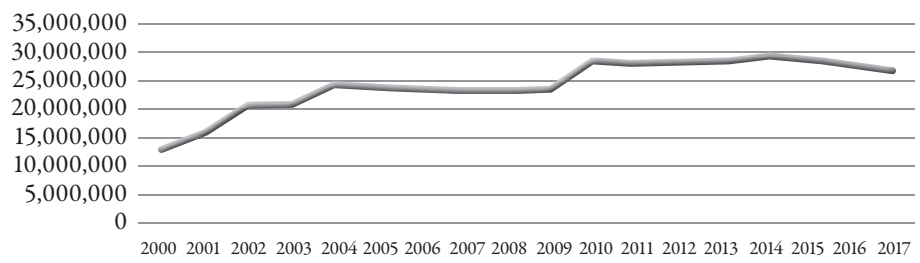

Fuente: Elaboración propia con base en Prospera, programa de inclusión social, 2019.

Ahora bien, hay varias investigaciones, que se han desarrollado entorno a la aplicación del programa social Prospera y la lucha contra la pobreza en México. Con respecto a la evolución de la pobreza, la gráfica 3 muestra que entre 2014 y 2017, la población pobre aumentó de $3.47 \%$, esto es, un incremento de $1,923,405$ de personas pobres.

Gráfica 3. Evolución de la pobreza 2008-2016.

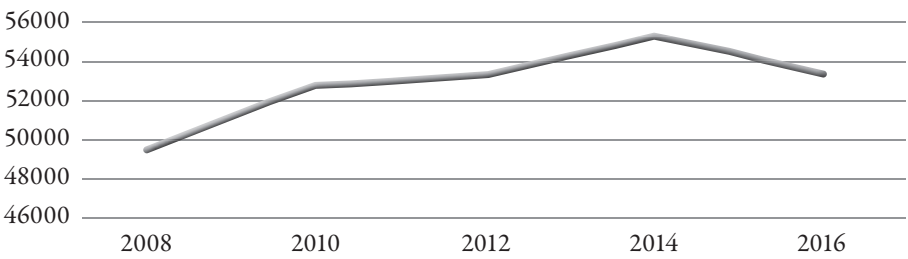

Fuente: Elaboración propia con base en las estimaciones del Coneval con base en el MCS-ENIGH 2008, 2010, 2012, 2014 y el MEC 2016 del MCS-ENIGH.

Enseguida, por un lado, un estudio de la Auditoria Superior de la Federación (2016) muestra que la cobertura de localidades 
con presencia de Prospera se incrementó en $7.4 \%$ en promedio anual, al pasar de $17.1 \%$ en 1998 a $58.0 \%$ en 2015 . Las localidades en las que está presente el programa son las que registran un mayor grado de marginación: las de muy alto grado de marginación con presencia del programa se incrementó en $4.3 \%$ en promedio anual, al pasar de $40.4 \%$ en 1998 a $82.7 \%$ en 2015; en las de alto grado de marginación, en $4.1 \%$, al pasar de $45.2 \%$ a $89.9 \%$, y en las de marginación media, en $13.4 \%$, al pasar de $10.0 \%$ a $84.4 \%$ en el mismo periodo.

Sólo que entre 2014 y 2017, el número de integrantes de las familias del programa PROSPERA de los programas infantiles disminuyó drásticamente, al pasar de 3,150,144 en 2013 a 1,763,442 en 2017, es decir, una caída de 56\% de integrantes de familias que reciben apoyo infantil vivir mejor entre los dos periodos. (Véase gráfica 4).

Gráfica 4. Integrantes de las familias del programa Prospera que reciben el apoyo infantil vivir mejor información correspondiente al cierre de ejercicio fiscal.

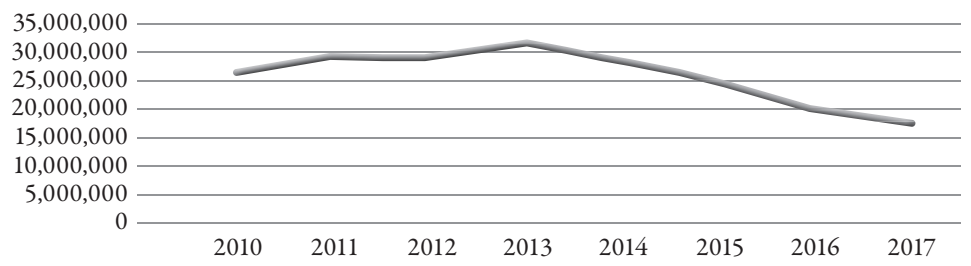

Fuente: Elaboración propia con base en Prospera, programa de inclusión social, 2019.

Por otro lado, de 1997 a 2015, Prospera incorporó al padrón activo de beneficiarios a 9,985.5 de familias, las cuales representaron el $87.2 \%$ de las $11,452.2$ identificadas como susceptibles de ser incorporadas, dadas sus condiciones socioeconómicas. Esta información analizada permite identificar que hubo años en los que la proporción de familias incorporadas fue mayor que las identificadas, lo cual obedece a que las familias a las que no se les pudo dar cabida se incorporaron al padrón base, y en cuanto existiera la disponibilidad, podían ser incorporadas, aunque fuera en ejercicios fiscales posteriores. (Auditoria Superior de la Federación, 2016). 
Después de 2014 se observa una disminución del padrón de familias beneficiarias, al pasar de 7,072,843 familias beneficiarias en 2014 a 6,584,061 en 2017. (Véase gráfica 5). La reducción de familias beneficiarias corresponde al $7 \%$.

Gráfica 5. Padrón de familias beneficiarias.

Cifras cierre de cuenta pública.

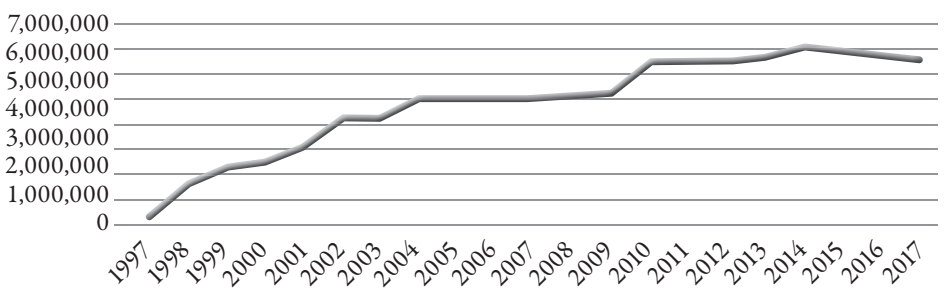

Fuente: Elaboración propia con base en Prospera, programa de inclusión social, 2019.

El programa Prospera ha traído mucho apoyo a las familias desfavorecidas, con carencias de alimentación, salud y otros. Sin embargo, estos esfuerzos no han sido constantes a lo largo de su aplicación, varios estudios muestran que el programa Prospera no ha alcanzado los objetivos planteados. En este caso, los datos arrojados de Prospera muestran que no se han dado los resultados esperados; pero también se entrevé que es preferible sostener y trasformar, que enfrentarse a una carga política por los beneficiarios del programa Prospera. ${ }^{22}$

Además, precisan que Prospera no permite aspirar a una cobertura total de las localidades del país, ya que la ampliación depende de la existencia y el desarrollo de infraestructura en educación y salud, lo cual es responsabilidad de esos sectores, sin que, en el marco del programa, se comprometa la focalización en la población en condición de pobreza que habitan en las localidades sin acceso a esos servicios, y, segundo, que esas localidades preci-

${ }_{22}$ Mendoza Magaña, Roberto. "Impactos del programa PROSPERA en el Estado de México (2012 y 2015)". Revista Electrónica del Centro de Estudios en Administración Pública de la Facultad de Ciencias Politicas y Sociales, 26 (2017), http://revistas. unam.mx/index.php/encrucijada/article/view/59703, (Consultado el 16 de abril de 2019). 
samente son las más marginadas, debido a que, dentro de las variables para determinar dicha condición, se ubica la educación. ${ }^{23}$

Esta situación genera una posible ruta encaminada al clientelismo mexicano. Cabría añadir el hecho de que, los datos del estudio no arrojarían información que represente un cubrimiento fehaciente en materia de pobreza o de carencia social por parte de Prospera, ya que intervendrían términos clientelares o de trasformación de la política pública que no justificarían del todo que los beneficiarios de dicho programa sean afines a esta condición social. ${ }^{24}$ Finalmente, un programa como Prospera solo trabaja a un nivel superficial de los problemas que les atañen a los mexiquenses puesto que es una respuesta al aquí y ahora sin un beneficio a posteriori.

También, en 2015, debido a la inaccesibilidad a los servicios educativos y de salud, el 15.9\% (31.6 miles) de las 199.3 miles de localidades del país no tuvo la posibilidad de ser atendido por Prospera, lo cual afectó de mayor manera a las localidades, no categorizadas en algún grado de marginación, en las que se observó que 31 de cada 100 localidades no tenían acceso a esos servicios; no obstante, de las 62.3 miles de localidades con alto grado de marginación, sólo el 1.6\% no tuvo disponibilidad de esos servicios, y de las de muy bajo, sólo el 3.6\% no dispuso de dichos servicios. ${ }^{25}$

Por ende, el programa Prospera ha brindado atención a cerca de 7 millones de familias, sosteniendo una cuarta parte del total de ingresos del décil más pobre de la población. Sin embar-

\footnotetext{
${ }^{23}$ Arenas, Erika. Teruel, Graciela y Flores, Karla Renata. "Resumen de Políticas Prospera Programa de Inclusión Social: Combate a la pobreza con perspectiva de género", Prospera-Sedesol. (2017), https:/www.gob.mx/cms/uploads/attachment/file/225888/Documento_de_pol_ticas_sobre_g_nero.pdf (Consultado el 25 de febrero de 2019).

${ }^{24}$ Mendoza Magaña, Roberto. "Impactos del programa PROSPERA en el Estado de México (2012 y 2015)”. Revista Electrónica del Centro de Estudios en Administración Pública de la Facultad de Ciencias Politicasy Sociales, 26 (2017). file:///D:/Usuarios/ad016301/ Downloads/59703-173333-1-PB\%20(3).pdf. (consultado el 23 abril de 2019).

${ }^{25}$ Auditoria Superior de la Federación. "Evaluación de la política pública de Prospera programa de inclusión social”, 2016, núm. 1575, Cámara de Diputados, https://www. asf.gob.mx/Trans/Informes/IR2015i/Documentos/Auditorias/2015_1575_a.pdf ((Consultado el 3 de febrero de 2020).
} 
go, el esfuerzo no ha traído el resultado esperado. No es sorprendente que el actual gobierno de Andres Manuel Lopez Obrador (AMLO) anunciará el fin de dicho programa de transferencias monetarias condicionadas que será reemplazado por un sencillo programa de becas, las famosas Becas para el Bienestar Benito Juárez que iniciaron el 28 de febrero del año $2019 .^{26}$

\section{Reflexiones Finales}

La economía mexicana no ha logrado la transición de pasar de una economía proteccionista a un actor de apertura comercial. Las multinacionales imponen y dominan el mercado. Esa situación de libre mercado genera mayor desigualdad entre la población y aumenta la pobreza. Frente a eso, el gobierno decidió implementar una serie de programas sociales con la finalidad de contrarrestar la pobreza, por lo que en 1997 nace el programa Prospera.

Con ese programa, se atendieron 115.6 miles de localidades, de las cuales el $73.4 \%$ correspondió a localidades con muy alto, alto y medio grado de marginación. El 6.0\%, a localidades con bajo y muy bajo grado, y el $20.6 \%$ a localidades sin clasificación, lo cual denota que, geográficamente, Prospera se ha acercado cada vez a comunidades más aisladas, ya que su cobertura se incrementó en 14.6 puntos porcentuales respecto de 2002 , y en 19.4 puntos porcentuales, respecto de 1998 según la Cámara de Diputados.

Lo que se observa es que, ${ }^{27}$ a pesar del discurso oficial, el programa sigue siendo el mismo, y las críticas hechas por el gobierno de Enrique Peña Nieto a Oportunidades son perfectamente aplicables a Prospera.

El gobierno actual de Andrés Manuel López Obrador tiene la difícil tarea de implementar programas que pudieran reducir de

\footnotetext{
${ }^{26}$ Secretaria de Gobernación. "Acuerdo por el que se emiten las Reglas de Operación de PROSPERA programa de Inclusión Social, para el ejercicio fiscal 2019”, 28/02/2019, Secretaría de Gobernación, https://www.dof.gob.mx/nota_detalle. php?codigo $=5551581 \&$ fecha $=28 / 02 / 2019$. (Consultado el 01 de enero 2020).

${ }^{27}$ Barajas Martínez, Gabriela. "Prospera, programa de inclusión social: ¿una nueva estrategia de atención a la pobreza en México?” Gestión y estrategia, 50, (2016), http://zaloamati.azc.uam.mx/bitstream/handle/11191/4931/Prospera-programa-de-inclusion-social-una-nueva-estrategia-de-atencion-a-la-pobreza-en-Mexico. pdf? sequence $=1 \&$ is Allowed $=y$ (Consultado 28-12- 2019).
} 
manera concreta y contundente la pobreza que cada vez va en aumento en nuestro país.

\section{Fuentes}

Aguilar Villanueva, Luis. Recepción y desarrollo de la disciplina de Política Pública en México. Un estudio introductorio. Sociológica, 19(54) (2004): 15-37.

Arenas, Erika; Teruel, Graciela y Flores, Karla Renata. "Resumen de Políticas PROSPERA Programa de Inclusión Social: Combate a la pobreza con perspectiva de género", Prospera-Sedesol. (2017). https://www.gob.mx/cms/uploads/attachment/ file/225888/Documento_de_pol_ticas_sobre_g_nero.pdf (Consultado el 25-2-2019).

Auditoria Superior de la Federación. "Evaluación de la política pública de Prospera programa de inclusión social", Auditoría Superior de la Federación, Cámara de Diputados. 2016, 1575, https:// www.asf.gob.mx/Trans/Informes/IR2015i/Documentos/Auditorias/2015_1575_a.pdf, (Consultado el 3-2-2019).

Banco Mundial, Pobreza. Banco Mundial. http://pubdocs.worldbank.org/en/571471424971740902/NT-Pobreza.pdf, (Consultado el 6-2-2019).

Banco Interamericano de Desarrollo, "BID lanza indicadores sociales y de equidad para medir avances y problemas en logro Objetivos de Desarrollo del Milenio", Banco Interamericano de Desarrollo. https://www.iadb.org/es/noticias/ comunicados-de-prensa/2005-10-28/bid-lanza-indicadores-sociales-y-de-equidad-para-medir-avances-y-problemas-en-logro-objetivos-de-desarrollo-del-milenio\%2c1188. html. (Consultado el 7-2-2019).

Barajas Martínez, Gabriela. Prospera, programa de inclusión social: ¿una nueva estrategia de atención a la pobreza en México?, 2016, Gestión y estrategia, 50(2016), http://zaloamati. azc.uam.mx/bitstream/handle/11191/4931/Prospera-programa-de-inclusion-social-una-nueva-estrategia-de-aten- 
82 Análisis del programa Prospera sobre la disminución de la pobreza en México

cion-a-la-pobreza-en-Mexico.pdf?sequence $=1$ \&isAllowed $=\mathrm{y}$ (Consultado el 28-12-2019).

Campos Vázquez, Raymundo y Monroy-Gómez Franco Luis. "La relación entre crecimiento económico y pobreza en México", Investigación Económica, 75, no. 298, (2016, octubre-diciembre), pp. 77-113, https://www.redalyc.org/pdf/601/60148183003.pdf

Cantú Herrera, Jorge Raúl y Chiatchoua, Cesaire (2018). Generación de políticas públicas en materia fiscal para la permanencia de las unidades económicas en México (2005-2017). Revista. ConCiencia. Vol.8, págs. 30-45. Consultado el 8 de junio de 2020 en: https://fup.edu.co/wp-content/uploads/2019/06/ conciencia_8_articulo_3.pdf.

CONEVAL (Consejo Nacional de Evaluación de la Política de Desarrollo Social), Estrategias de coordinación interinstitucional para la política de desarrollo social en México, 2019. CONEVAL, https://www.coneval.org.mx/Evaluacion/ IEPSM/Documents/Documento-completo-40-A-de-estrategias.pdf. (Consultado el 13-11-2018).

Fernández, Andrés. Martínez, Rodrigo. Carrasco, Ignacio y Palma, Amalia. "Impacto social y económico de la malnutrición. Un modelo de análisis y estudio piloto en Chile, el Ecuador y México”. Programa Mundial de Alimentos, CEPAL, Naciones Unidas. 2017.

Jiménez Guzmán, Rodolfo. Las políticas públicas, gerencia y gestión pública moderna, panaceas de racionalidad y eficiencia, o nuevas tecnologías de gobierno. UAM: Revista casa del tiempo. México: 1999.

Mendoza Magaña, R. Impactos del programa PROSPERA en el Estado de México (2012 y 2015). Revista Electrónica del Centro de Estudios en Administración Pública de la Facultad de Ciencias Políticas y Sociales, 26(2017) http://revistas.unam. $\mathrm{mx} /$ index.php/encrucijada/article/view/59703. (Consultado el 16-04-2019)

Natanson, José. ¿Qué es, en realidad, una política de Estado? Página 12, (29 de junio de 2008 Recuperado de: https://www. 
pagina12.com.ar/diario/elpais/1-106918-2008-06-29.html. (Consultado el 12-10-2019)

Ortiz, Isabel. Política Social. 2007. Documento de las Estrategias nacionales de Desarrollo. Naciones Unidas. https://esa. un.org/techcoop/documents/socialpolicy_spanish.pdf (Consultado el 20-2-2020).

Romero, Alberto. "Cómo crear políticas de Estado". Perfil. com. http:// www.perfil.com/contenidos/2010/08/21/noticia_0034.html (Consultado el 6-5-2011).

Rulli, Jorge. "Producir soja como política de Estado". Ecoportal. net. http://www.ecoportal.net/Temas_Especiales/Transgénicos/Producir_soja_como_politica_de_Estado. (Consultado el 8-1-2020).

Secretaria de Gobernación. "Acuerdo por el que se emiten las Reglas de Operación de PROSPERA programa de Inclusión Social, para el ejercicio fiscal 2018", Secretaría de Gobernación https://dof.gob.mx/nota_detalle.php?codigo $=5509738 \&$ fecha $=29 / 12 / 2017$ (Consultado el 1-1-2020).

Secretaria de Gobernación. "Acuerdo por el que se emiten las Reglas de Operación de PROSPERA programa de Inclusión Social, para el ejercicio fiscal 2019", Secretaría de Gobernación https://www.dof.gob.mx/nota_detalle.php?codigo $=5551581 \&$ fecha $=28 / 02 / 2019$ (Consultado el 2-1-2020).

Székely Pardo, Miguel y Díaz Ortega, Araceli, "Pobreza alimentaria y desarrollo en México". Trimestre Económico, 81(321) (2014): 43-105.

Tejerina, Luis y Villa, Juan Miguel. América Latina y el Caribe deberían aprovechar los datos masivos para medir la pobreza. Impacto. https://blogs.iadb.org/efectividad-desarrollo/es/ america-latina-deberia-aprovechar-los-datos-masivos-para-medir-la-pobreza/ (Consultado el 7-2-2019).

World Bank Group Poverty. "Pobreza”. World Bank Group. http://pubdocs.worldbank.org/en/571471424971740902/ NT-Pobreza.pdf. (Consultado el 7-10-2019). 
